\title{
Planetesimal formation near the snow line in MRI-driven turbulent protoplanetary disks
}

\author{
F. Brauer, Th. Henning, and C. P. Dullemond
}

\author{
Max-Planck-Institut für Astronomie, Königstuhl 17, 69117 Heidelberg, Germany \\ e-mail: brauer@mpia.de
}

Received 14 March 2008 / Accepted 6 June 2008

ABSTRACT

\begin{abstract}
The formation of planetesimals in protoplanetary disks due to collisional sticking of smaller dust aggregates has to face at least two severe obstacles, namely the rapid loss of material due to radial inward drift and particle fragmentation due to destructive collisions. We present a scenario to circumvent these two hurdles. Our dust evolution model involves two main mechanisms. First, we consider a disk with a dead zone. In an almost laminar region close to the midplane, the relative velocities of the turbulent particles are comparatively small, which decreases the probability of destructive particle collisions. Second, turbulence is not the only source of violent relative particle velocities, because high radial drift speeds can also lead to boulder fragmentation. For this reason, we focus additionally on the snow line. Evaporation fronts can be associated with gas pressure maxima in which radial drift basically vanishes. This implies that particle fragmentation becomes even less likely. Our simulation results suggest that particles can overcome the fragmentation barrier. We find that boulders of several $10^{2} \mathrm{~m}$ can form within only a few thousand years.
\end{abstract}

Key words. accretion, accretion disks - circumstellar matter - stars: formation - stars: pre-main-sequence planetary systems: protoplanetary disks

\section{Introduction}

The process of planetary formation is widely accepted to be initiated in circumstellar disks by the collisional coagulation of dust grains to larger objects (Henning et al. 2006; Natta et al. 2007). Evidence of grain growth to particle sizes beyond those typically observed in the interstellar medium is provided by mid-infrared spectroscopy of disks around young stars (Bouwman et al. 2001; van Boekel et al. 2003; Apai et al. 2004; Kessler-Silacci et al. 2007; Sicilia-Aguilar et al. 2007). Analyses of millimeter interferometry data of disks implies that large populations of dust grains exist with particle radii up to several millimeters (Testi et al. 2003; Wilner et al. 2005; Rodmann et al. 2006).

Models of dust particle growth in protoplanetary disks predict coagulation, which supports the idea of planetesimal formation by particle hit and stick mechanisms. Numerical calculations support the formation of $\mathrm{cm}$-sized particles within a few $10^{3}$ orbital timescales in the inner parts of the disk (Weidenschilling 1980). After particles have grown to larger sizes, they can also be affected by other processes described by alternative planet formation scenarios. For example, large grains experience vertical settling, which involves the formation of a dense midplane dust layer (Garaud \& Lin 2004). This layer can be affected by gravitational instability (Goldreich \& Ward 1973; Weidenschilling 1979; Youdin \& Shu 2002; Schräpler \& Henning 2004; Garaud \& Lin 2004) - an issue that is still highly debated. Due to the overwhelming amount of observational data for protoplanetary disks available, models of dust particle growth have attracted more attention (Tanaka et al. 2005; Dullemond \& Dominik 2005; Nomura \& Nakagawa 2006; D'Alessio et al. 2006; Ormel et al. 2007; Ciesla 2007). All of these particle evolution models can explain observational disk features such as, for example, the group I/II disk classification
(Meeus et al. 2001; Dullemond \& Dominik 2004) or the disappearance of infrared excess in the spectra of disks with ages higher than a few Myrs (Haisch et al. 2001; Carpenter et al. 2005), although, there are still many open observational riddles that these models will hopefully answer in the near future.

Models of protoplanetary disks do not only help to interpret observational data; they also identify the serious obstacles to planetesimal formation (Youdin 2004; Dominik et al. 2007). One of these obstacles is particle fragmentation. Due to the high particle relative velocities in disks that can reach 100 m/s (Weidenschilling 1977; Ormel \& Cuzzi 2007), particle collisions lead to particle destruction instead of particle growth (Blum \& Wurm 2000; Brauer et al. 2008; Johansen et al. 2008). Depending on the disk model, dust particle growth is inhibited significantly by high-speed impacts of particles around a meter size. Even for times as long as $1 \mathrm{Myr}$, solid particles are unable to overcome the fragmentation barrier and the formation of planetesimals as precursors for Earth-like or Jovian planets, therefore, poses a major problem. Despite this theoretically predicted inability for planetesimals to form, the orbital decay of dust particles can, nevertheless, reproduce the observed evolution in the spectral energy distribution (Haisch et al. 2001; Carpenter et al. 2005). The presence of a growth barrier is, therefore, not in contradiction with observations.

Several mechanisms in protoplanetary disks can produce planetesimal formation. Johansen et al. (2007) showed that the non-linear feedback of dust onto gas can lead to the rapid formation of gravitationally bound clumps of dust, which subsequently form Ceres-size bodies. The dust particles, however, must have already grown to some meters in size before this scenario can take place. Another possibility for solving the formation problem is particle trapping in gas pressure maxima 
(Barge \& Sommeria 1995; Klahr \& Henning 1997). In gas pressure bumps, relative particle velocities are substantially lower and, hence, the collision between large bodies is more likely to lead to particle growth than to particle disruption.

Interesting means of gas pressure maxima and dust particle trapping was presented by Kretke \& Lin (2007). This particle retention mechanism requires the presence of an evaporation front, for example the snow line, which acts in the following way. As we travel through the snow line in a direction away from the central star, the dust-to-gas ratio increases suddenly. This jump in dust density affects the strength of the magneto-rotational turbulence, since the amount of free electrons in the disks strongly depends on the dust density. With increasing dust density, the amount of turbulence in the disk decreases (Sano et al. 2000; Ilgner \& Nelson 2006). We assume a constant mass accretion rate throughout the disk. The gas surface density then has to be higher in low than in high disk turbulent regions for the gas mass accretion to remain at a similar value. The sudden increase in the dust density could therefore also reproduce a sudden increase in gas densities. For certain accretion rates, Kretke \& Lin (2007) found the occurrence of a local gas density bump in which solid particles tend to accumulate. In their simulations, dust particle retention reproduced very high surface dust densities of the order of several $10^{3} \mathrm{~g} / \mathrm{cm}^{2}$, which again raises concern about gravitational instability.

However, even for a particle retention mechanism similar to that described above, the growth of solid material towards larger sizes remains an open issue. Gas pressure maxima may decrease relative radial particle velocities triggering coagulation; the most severe reason for violent particle fragmentation is not however radial drift but the turbulent nature of the protostellar disk itself. Relative turbulent particle velocities are approximately $10 \mathrm{~m} / \mathrm{s}$ (Ormel \& Cuzzi 2007; Völk et al. 1980) and particle sticking at these high speeds is extremely unlikely. Nevertheless, Ciesla (2007) found that particles can grow to several $10 \mathrm{~m}$ in radius if we consider layered MRI active disks. Under certain circumstances, MRI is only active in the upper layers of the disk, while the disk midplane is almost laminar. Since most of the larger grains are located around the midplane where the disk is quiescent, dust growth is not inhibited by the high speed collisions that produce particle fragmentation.

We combine three ingredients for a planetesimal formation model. We consider dust particle growth, particle fragmentation and radial motion (Brauer et al. 2008) around the snow line (Kretke \& Lin 2007) in a layered MRI active protoplanetary disk (Ciesla 2007). The inclusion of a snow line in our simulations provides a particle retention mechanism which almost erases the radial drift velocities favouring particle growth. We adopt a layered MRI-driven disk to circumvent particle fragmentation in the midplane of the disk due to turbulent motions of the gas. We investigate whether solid particles can overcome the fragmentation barrier and produce larger objects, which represent the possible precursors to the planets. We study the influence of two parameters, namely the gas accretion rate $\dot{M}_{\text {acc }}$ and the critical threshold velocity for fragmentation $v_{\mathrm{f}}$.

\section{Model}

We assume a background gas disk which is in a steady state and, hence, does not change with time. The calculation of the gas densities around the snow line are described in detail in Kretke $\&$ Lin (2007). We adopt all of the parameter values presented in that paper apart from the residual turbulence value around the midplane $\alpha_{0}$, which we set to be $10^{-5}$ corresponding to

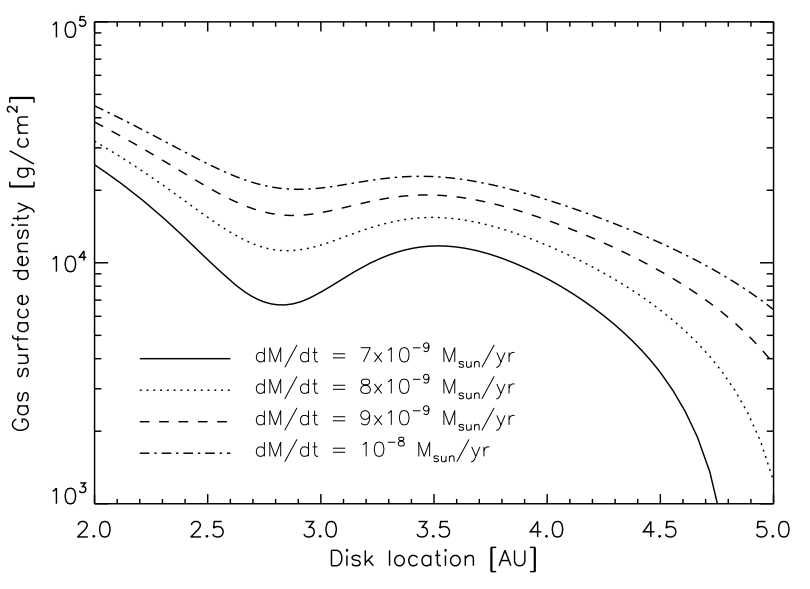

Fig. 1. The surface gas densities for different gas accretion rates which we will use in our simulations as calculated by Kretke \& Lin (2007) and discussed in Sect. 2. The snow line is located at $3 \mathrm{AU}$.

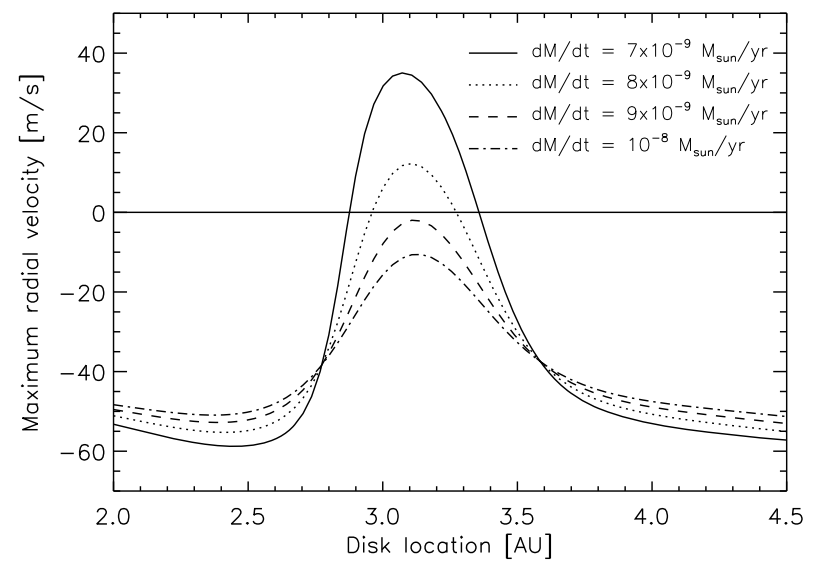

Fig. 2. Maximum radial drift velocity $v_{\mathrm{N}}$ for different gas accretion rates as discussed in Sect. 2. The horizontal line indicates zero radial drift. Outward drift occurs for the two accretion rates $7 \times 10^{-9}$ and $8 \times 10^{-9} M_{\text {sun }} / y$. Hence, we expect dust grain retention for these two values. Note as well, that for these two accretion rates, there are two stagnation points in which the radial drift speed vanishes.

self-induced turbulence (Weidenschilling 1979; Weidenschilling \& Cuzzi 1993). The water evaporation front, which is the important element in our model, is located at 3 AU (Lecar et al. 2006). The gas surface densities that we adopt in our simulations are shown in Fig. 1 for different accretion rates $\dot{M}_{\text {acc }}$.

We focus on the dust component of the disk. We include the radial drift motion of solids in our model. The maximum radial inward drift velocity is given by

$v_{\mathrm{N}}=\frac{\partial_{\mathrm{r}} P_{\mathrm{g}}}{2 \rho_{\mathrm{g}} \Omega_{\mathrm{k}}}$.

For different accretion rates, this quantity is shown in Fig. 2 as a function of disk location. The equations for the gas pressure $P_{\mathrm{g}}$ and the Kepler frequency $\Omega_{\mathrm{k}}$ are given in Brauer et al. (2008). From this maximum radial drift velocity, we can calculate the actual radial drift speed of a particle of a certain size and solve the continuity equation in the radial direction for all dust particle species. Radial diffusion due to turbulent mixing is in addition included in the model.

Figure 2 shows, that for sufficiently small accretion rates, grain retention will occur around the snow line close to $3 \mathrm{AU}$ inside the disk. For dust particle growth, it is even more important 


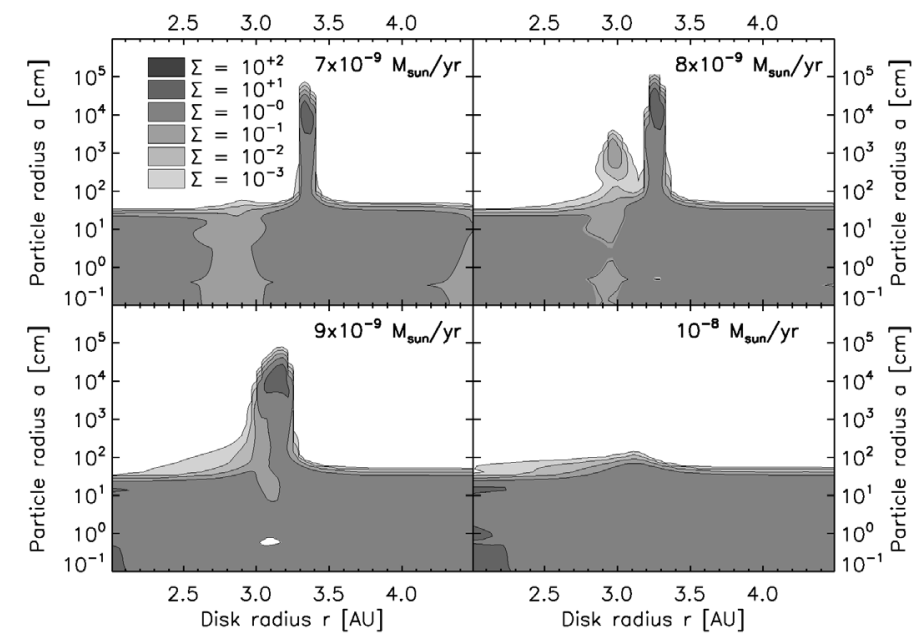

Fig. 3. The particle distribution after 1800 yrs of disk evolution as discussed in Sect. 3. Shown is a contour plot of the surface dust density as a function of disk location and particle radius for four different accretion rates. The figure indicates that particles break through the fragmentation barrier if the accretion rate is not too high. The critical fragmentation velocity is $10 \mathrm{~m} / \mathrm{s}$ in this simulation.

to note that there are two radial drift stagnation points, i.e. disk locations at which the radial drift completely vanishes. Relative particle speeds at these radii are too low to induce destructive particle collisions. While solid particles move away from the inner diverging point at $\sim 2.9 \mathrm{AU}$, the dust tends to accumulate in the outer converging point at $\sim 3.3 \mathrm{AU}$. It is also interesting to note here, that the azimuthal gas flow becomes Keplerian at these stagnation points. The differential azimuthal speed between the dust and gas vanishes, which implies that self-induced turbulence is stablized regardless on how thin the dust layer becomes.

We consider that the disk has a vertical structure. The vertical dust distribution is determined by turbulent diffusion and particle settling towards the midplane (Dubrulle et al. 1995; Schräpler \& Henning 2004). We solve the sedimentation/diffusion equation with a semi-analytical model, assuming that the particle distribution is in equilibrium between particle settling and turbulent mixing. We consider a dead zone around the midplane in which almost no turbulence is present. In this region, we adopt an $\alpha$-value of $10^{-5}$. In the MRI active regions on the surface of the disk, we assume an $\alpha$ parameter of $1.8 \times 10^{-2}$ (Sano et al. 1998). The amount of gas and dust mass that is MRI active/inactive is provided in Kretke \& Lin (2007). Little turbulence around the midplane involves small relative turbulent velocities (Ormel \& Cuzzi 2007). Hence, in the radial drift stagnation points, all relative velocities are low and we may expect dust particles to grow to larger sizes and break through the fragmentation barrier in the midplane of the disk.

At every point in the disk, we allow dust particles to coagulate and grow to larger sizes or suffer fragmentation due to high speed collisions. The parameter that distinguishes between these two cases is the collision velocity of the particles. If this quantity is higher than a critical threshold velocity $v_{\mathrm{f}}$, fragmentation occurs. Apart from the accretion rate $\dot{M}_{\text {acc }}$, the threshold fragmentation velocity is the second parameter that we consider. In the simulations, we assume five different sources of relative particle velocities in the disk that generate dust growth, namely Brownian motion, differential settling, relative turbulent velocities, and relative radial and azimuthal velocities. All of these sources are explained extensively in Brauer et al. (2008), which

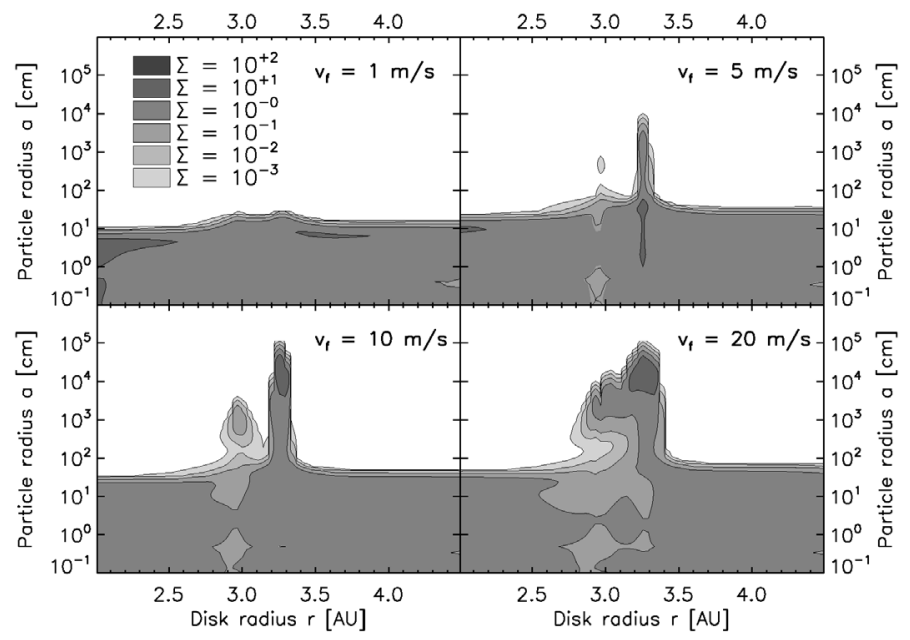

Fig. 4. As Fig. 3, but now for four different critical theshold fragmentation velocities. The figure indicates that dust particles can break through the fragmentation barrier if the theshold fragmentation velocity is at least $5 \mathrm{~m} / \mathrm{s}$. In this simulation, we adopted an accretion rate of $\dot{M}_{\text {acc }}=8 \times 10^{-9} M_{\text {sun }} / \mathrm{yr}$.

also describes the numerical schemes adopted to solve the coagulation equation (i.e. Smoluchowski equation), the outcome of fragmentation, and the effect of cratering that is also included in the model.

In the final simulations completed by Brauer et al. (2008), particles were always smaller than the mean free path of the gas at any point in the disk. This is not the case here due to the very high gas densities. This implies that we must consider a different drag force regime, namely the Stokes regime instead of the Epstein regime. We implemented this regime into our model to account for the high gas densities (Weidenschilling 1977).

\section{Results}

Figure 3 shows the particle distribution after 1800 yrs of disk evolution for different accretion rates. In this simulation, we adopted a critical threshold fragmentation velocity of $10 \mathrm{~m} / \mathrm{s}$. This plot indicates that particles can grow to some $10^{2} \mathrm{~m}$ in size around the ice evaporation front subject to the condition that the gas accretion rate is not too high. It also shows that fragmentation inhibits particle growth towards m-sized particles in other disk regions, for example around 4 AU. To unveil the importance of collective effects at the snow line, we estimate the dust-to-gas ratio in the midplane of the disk. The vertically integrated ratio in the case of $\dot{M}=9 \times 10^{-9} M_{\text {sun }} / \mathrm{yr}$ is of the order $\epsilon_{0}=10 / 10^{4}=10^{-3}$, as inferred from Figs. 1 and 3. The dust-to-gas ratio in the midplane is then at least $\epsilon_{\text {mid }} \approx \epsilon_{0} \sqrt{\mathrm{St} / \alpha} \approx 10^{-3} \sqrt{10^{3} / 10^{-5}}=10>1$, which means that collective effects play a non-negligible role. Since collective effects strongly influence the radial drift behaviour of the dust, further investigation of this issue is certainly required.

The accretion rate of a protoplanetary disk decreases with time. Therefore, Fig. 3 illustrates the advantage of our planetesimal formation mechanism at different stages of disk evolution. In the early stages, the gas surface densities are not significantly affected by the sharp decrease in dust-to-gas ratio at the snow line because the accretion rate is too high. Hence, we do not find that particles grow to become very large objects in this case. With decreasing accretion rate, the surface gas density becomes 
more and more affected by the snow line producing the formation of large boulders as shown in Fig. 3. At this stage of disk evolution, planetesimal formation could occur. At later stages, the surface gas densities will have declined towards a level at which the entire disk becomes MRI active and the mechanism is unable to operate.

The dust particle distribution after 1800 yrs, but now for different criticial fragmentation velocities, is shown in Fig. 4. In this calculation, we considered an accretion rate of $\dot{M}_{\text {acc }}=8 \times 10^{-9} M_{\text {sun }} / \mathrm{yr}$. This plot indicates that dust particles can overcome the fragmentation barrier and grow to nearly $\mathrm{km}$-size if the critical threshold velocity is at least $5 \mathrm{~m} / \mathrm{s}$. For the case $v_{\mathrm{f}}=1 \mathrm{~m} / \mathrm{s}$, we do not find particles that are able to grow to larger than a meter in radius. This is due to the fact that relative turbulent velocities in our quiescent midplane are still of the order of several m/s. Even with a low residual turbulent $\alpha_{0}$-value of $10^{-6}$, relative particle velocities would hardly decrease to below this value.

The residual turbulent $\alpha_{0}$-value around the midplane can be shifted to higher values due to the occurrence of turbulent convection. Klahr \& Henning (1997) suggest turbulent gas speeds of $\sim 0.02 c_{\mathrm{s}}$, which correspond to velocities of $\sim 16 \mathrm{~m} / \mathrm{s}$ at $1 \mathrm{AU}$ and an $\alpha$-value of $\sim 10^{-4}$. Hence, if convection is a driving source of turbulence in the disk then the results of this Letter might change significantly. Turner et al. (2007) showed that free charges can be mixed into the disk interior, producing a slight coupling between the midplane gas and the magnetic fields. This may generate an active turbulent midlane layer, which could trigger particle fragmentation. Further investigation of the influence of each of these effects is imperative.

\section{Summary and conclusions}

Even though particle fragmentation by high speed collisions is a severe obstacle to the formation of planetesimals, we have shown that dust particle growth to the size of enormous boulders is possible under certain circumstances. If we consider dust particle coagulation in the presence of an evaporation front, solid particles grow to almost km-sizes within only a few thousand years. The protoplanetary disk must also contain a dead zone in which turbulence - the main source of relative particle velocities - is almost entirely absent. Our conclusion is that planetesimal formation due to dust particle agglomeration is a possible mechanism to form large bodies in protoplanetary disks.

Acknowledgements. We wish to thank A. Johansen and H. Klahr for helpful discussions. We also thank the anonymous referee for useful comments that helped us to improve this Letter.

\section{References}

Apai, D., Pascucci, I., Sterzik, M. F., et al. 2004, A\&A, 426, L53 Barge, P., \& Sommeria, J. 1995, A\&A, 295, L1

Blum, J., \& Wurm, G. 2000, Icarus, 143, 138

Bouwman, J., Meeus, G., de Koter, A., et al. 2001, A\&A, 375, 950

Brauer, F., Dullemond, C. P., \& Henning, T. 2008, A\&A, 480, 859

Carpenter, J. M., Wolf, S., Schreyer, K., Launhardt, R., \& Henning, T. 2005, AJ, 129,1049

Ciesla, F. J. 2007, ApJL, 654, L159

D’Alessio, P., Calvet, N., Hartmann, L., Franco-Hernández, R., \& Servín, H. 2006, ApJ, 638, 314

Dominik, C., Blum, J., Cuzzi, J. N., \& Wurm, G. 2007, in Protostars and Planets V, ed. B. Reipurth, D. Jewitt, \& K. Keil, 783

Dubrulle, B., Morfill, G., \& Sterzik, M. 1995, Icarus, 114, 237

Dullemond, C. P., \& Dominik, C. 2004, A\&A, 417, 159

Dullemond, C. P., \& Dominik, C. 2005, A\&A, 434, 971

Garaud, P., \& Lin, D. N. C. 2004, ApJ, 608, 1050

Goldreich, P. \& Ward, W. R. 1973, ApJ, 183, 1051

Haisch, Jr., K. E., Lada, E. A., \& Lada, C. J. 2001, ApJ, 553, L153

Henning, T., Dullemond, C. P., Wolf, S., \& Dominik, C. 2006, Dust coagulation in protoplanetary disks (Planet Formation), 112

Ilgner, M., \& Nelson, R. P. 2006, A\&A, 445, 205

Johansen, A., Oishi, J. S., Low, M.-M. M., et al. 2007, Nature, 448, 1022

Johansen, A., Brauer, F., Dullemond, C., Klahr, H., \& Henning, T. 2008, ArXiv e-prints, 802

Kessler-Silacci, J. E., Dullemond, C. P., Augereau, J.-C., et al. 2007, ApJ, 659, 680

Klahr, H. H., \& Henning, T. 1997, Icarus, 128, 213

Kretke, K. A., \& Lin, D. N. C. 2007, ApJ, 664, L55

Lecar, M., Podolak, M., Sasselov, D., \& Chiang, E. 2006, ApJ, 640, 1115

Meeus, G., Waters, L. B. F. M., Bouwman, J., et al. 2001, A\&A, 365, 476

Natta, A., Testi, L., Calvet, N., et al. 2007, in Protostars and Planets V, ed. B. Reipurth, D. Jewitt, \& K. Keil, 767

Nomura, H., \& Nakagawa, Y. 2006, ApJ, 640, 1099

Ormel, C. W., \& Cuzzi, J. N. 2007, A\&A, 466, 413

Ormel, C. W., Spaans, M., \& Tielens, A. G. G. M. 2007, A\&A, 461, 215

Rodmann, J., Henning, T., Chandler, C. J., Mundy, L. G., \& Wilner, D. J. 2006, A\&A, 446, 211

Sano, T., Inutsuka, S.-I., \& Miyama, S. M. 1998, ApJ, 506, L57

Sano, T., Miyama, S. M., Umebayashi, T., \& Nakano, T. 2000, ApJ, 543, 486

Schräpler, R., \& Henning, T. 2004, ApJ, 614, 960

Sicilia-Aguilar, A., Hartmann, L. W., Watson, D., et al. 2007, ApJ, 659, 1637

Tanaka, H., Himeno, Y., \& Ida, S. 2005, ApJ, 625, 414

Testi, L., Natta, A., Shepherd, D. S., \& Wilner, D. J. 2003, A\&A, 403, 323

Turner, N. J., Sano, T., \& Dziourkevitch, N. 2007, ApJ, 659, 729

van Boekel, R., Waters, L. B. F. M., Dominik, C., et al. 2003, A\&A, 400, L21

Völk, H. J., Morfill, G. E., Roeser, S., \& Jones, F. C. 1980, A\&A, 85, 316

Weidenschilling, S. J. 1977, MNRAS, 180, 57

Weidenschilling, S. J. 1979, BAAS, 11, 552

Weidenschilling, S. J. 1980, Icarus, 44, 172

Weidenschilling, S. J., \& Cuzzi, J. N. 1993, in Protostars and Planets III, ed. E. H. Levy, \& J. I. Lunine, 1031

Wilner, D. J., D’Alessio, P., Calvet, N., Claussen, M. J., \& Hartmann, L. 2005, ApJ, 626, L109

Youdin, A. N. 2004, in ASP Conf. Ser., 323, 319

Youdin, A. N., \& Shu, F. H. 2002, ApJ, 580, 494 